KYUNGPOOK Math. J. 52(2012), 413-431

http://dx.doi.org/10.5666/KMJ.2012.52.4.413

\title{
Conditional Integral Transforms on a Function Space
}

\author{
Dong Hyun CHO \\ Department of Mathematics, Kyonggi University, Suwon, 443-760, Korea \\ e-mail : j94385@kyonggi.ac.kr
}

ABstract. Let $C^{r}[0, t]$ be the function space of the vector-valued continuous paths $x:[0, t] \rightarrow \mathbb{R}^{r}$ and define $X_{t}: C^{r}[0, t] \rightarrow \mathbb{R}^{(n+1) r}$ and $Y_{t}: C^{r}[0, t] \rightarrow \mathbb{R}^{n r}$ by $X_{t}(x)=\left(x\left(t_{0}\right), x\left(t_{1}\right), \cdots, x\left(t_{n-1}\right), x\left(t_{n}\right)\right)$ and $Y_{t}(x)=\left(x\left(t_{0}\right), x\left(t_{1}\right), \cdots, x\left(t_{n-1}\right)\right)$, respectively, where $0=t_{0}<t_{1}<\cdots<t_{n}=t$. In the present paper, using two simple formulas for the conditional expectations over $C^{r}[0, t]$ with the conditioning functions $X_{t}$ and $Y_{t}$, we establish evaluation formulas for the analogue of the conditional analytic Fourier-Feynman transform for the function of the form

$$
\exp \left\{\int_{0}^{t} \theta(s, x(s)) d \eta(s)\right\} \psi(x(t)), \quad x \in C^{r}[0, t]
$$

where $\eta$ is a complex Borel measure on $[0, t]$ and both $\theta(s, \cdot)$ and $\psi$ are the Fourier-Stieltjes transforms of the complex Borel measures on $\mathbb{R}^{r}$.

\section{Introduction and an analogue of the r-dimensional Wiener space}

Let $C_{0}[0, t]$ be the space of real-valued continuous functions $x$ on $[0, t]$ with $x(0)=0$. It is well known that the space $C_{0}[0, t]$ is equipped with the Wiener measure which is a probability measure. On the space, Yeh $[10,11,12]$ introduced an inversion formula that a conditional expectation can be found by a Fouriertransform. In [2], the author and his co-authors introduced a simple formula for the conditional Wiener integrals over $C_{0}(\mathbb{B})$, the space of the abstract Wiener space $\mathbb{B}$-valued continuous functions which vanish at 0 . Using the formula, they established various evaluation formulas for the conditional analytic Wiener and Feynman integrals of the functionals on $C_{0}(\mathbb{B})$ in a certain Banach algebra which corresponds to the Cameron and Storvick's Banach algebra $\mathcal{S}^{\prime \prime}$ [1]. In [3], the author evaluated conditional analytic Fourier-Feynman transform for functional of the form

$$
\exp \left\{\int_{0}^{t} \theta(s, x(s)) d \zeta(s)\right\} \phi(x(t)), \quad x \in C_{0}(\mathbb{B})
$$

Received March 3, 2010; accepted September 23, 2011.

2010 Mathematics Subject Classification: 28C20.

Key words and phrases: Analogue of Wiener measure, Conditional Feynman integral, Conditional Fourier-Feynman transform, Conditional Wiener integral, Simple formula for conditional Wiener integral. 
where $\zeta$ is a complex Borel measure on $[0, t]$ and both $\theta(s, \cdot)$ and $\phi$ are the FourierStieltjes transforms of the complex Borel measures on the real separable Hilbert space embedded in $\mathbb{B}$. Note that $\theta(s, \cdot)$ and $\phi$ are defined on $\mathbb{B}$, the abstract Wiener space [8].

On the other hand, let $C[0, t]$ denote the space of real-valued continuous functions on the interval $[0, t]$. Ryu and $\operatorname{Im}[6,9]$ introduced a probability measure $w_{\varphi}$ on $(C[0, t], \mathcal{B}(C[0, t]))$, where $\mathcal{B}(C[0, t])$ denotes the Borel $\sigma$-algebra on $C[0, t]$ and $\varphi$ is a probability distribution on $(\mathbb{R}, \mathcal{B}(\mathbb{R}))$. This measure space is a generalization of the Wiener space $C_{0}[0, t]$. In the Wiener space, every path $x$ starts at the origin, that is, $x(0)=0$. If the paths $x$ start at any points, that is, if $x \in C[0, t]$, certain properties on $C_{0}[0, t]$ can not hold or some of them should be modified. Fortunately, in $[4,5]$, the author could derive two simple formulas for the conditional $w_{\varphi}$-integrals of the functions on $C[0, t]$ with the vector-valued conditioning functions $X: C[0, t] \rightarrow \mathbb{R}^{n+1}$ and $Y: C[0, t] \rightarrow \mathbb{R}^{n}$ given by $X(x)=\left(x\left(t_{0}\right), x\left(t_{1}\right), \cdots, x\left(t_{n}\right)\right)$ and $Y(x)=\left(x\left(t_{0}\right), x\left(t_{1}\right), \cdots, x\left(t_{n-1}\right)\right)$, where $0=t_{0}<t_{1}<\cdots<t_{n-1}<t_{n}=t$ is a partition of $[0, t]$. These formulas express the conditional $w_{\varphi}$-integrals directly in terms of the non-conditional $w_{\varphi}$-integrals.

Let $C^{r}[0, t]$ be the product space of $C[0, t]$ and define $X_{t}: C^{r}[0, t] \rightarrow \mathbb{R}^{(n+1) r}$ and $Y_{t}: C^{r}[0, t] \rightarrow \mathbb{R}^{n r}$ by $X_{t}(x)=\left(x\left(t_{0}\right), x\left(t_{1}\right), \cdots, x\left(t_{n-1}\right), x\left(t_{n}\right)\right)$ and $Y_{t}(x)=$ $\left(x\left(t_{0}\right), x\left(t_{1}\right), \cdots, x\left(t_{n-1}\right)\right)$. In the present paper, with the conditioning functions $X_{t}$ and $Y_{t}$, we introduce two simple formulas for the conditional expectations over $C^{r}[0, t]$, an $r$-dimensional analogue of Wiener space. We then establish evaluation formulas for an analogue of the conditional analytic Fourier-Feynman transform for the function of the form

$$
\exp \left\{\int_{0}^{t} \theta(s, x(s)) d \eta(s)\right\} \psi(x(t)), \quad x \in C^{r}[0, t]
$$

where $\eta$ is a complex Borel measure on $[0, t]$ and both $\theta(s, \cdot)$ and $\psi$ are the FourierStieltjes transforms of the complex Borel measures on $\mathbb{R}^{r}$.

Throughout this paper, let $\mathbb{C}$ and $\mathbb{C}_{+}$denote the sets of complex numbers and complex numbers with positive real parts, respectively.

Now, we introduce the probability measure $w_{\varphi}$ on $(C[0, t], \mathcal{B}(C[0, t]))$.

For a positive real $t$, let $C=C[0, t]$ be the space of all real-valued continuous functions on the closed interval $[0, t]$ with the supremum norm. For $\vec{t}=\left(t_{0}, t_{1}, \cdots, t_{n}\right)$ with $0=t_{0}<t_{1}<\cdots<t_{n} \leq t$, let $J_{\vec{t}}: C[0, t] \rightarrow \mathbb{R}^{n+1}$ be the function given by

$$
J_{\vec{t}}(x)=\left(x\left(t_{0}\right), x\left(t_{1}\right), \cdots, x\left(t_{n}\right)\right) .
$$

For $B_{j}(j=0,1, \cdots, n)$ in $\mathcal{B}(\mathbb{R})$, the subset $J_{\vec{t}}^{-1}\left(\prod_{j=0}^{n} B_{j}\right)$ of $C[0, t]$ is called an interval and let $\mathcal{J}$ be the set of all such intervals. For a probability measure $\varphi$ on 
$(\mathbb{R}, \mathcal{B}(\mathbb{R}))$, let

$$
\begin{aligned}
m_{\varphi}\left(J_{\vec{t}}^{-1}\left(\prod_{j=0}^{n} B_{j}\right)\right)= & {\left[\prod_{j=1}^{n} \frac{1}{2 \pi\left(t_{j}-t_{j-1}\right)}\right]^{\frac{1}{2}} \int_{B_{0}} \int_{\prod_{j=1}^{n} B_{j}} } \\
& \exp \left\{-\frac{1}{2} \sum_{j=1}^{n} \frac{\left(u_{j}-u_{j-1}\right)^{2}}{t_{j}-t_{j-1}}\right\} d\left(u_{1}, \cdots, u_{n}\right) d \varphi\left(u_{0}\right) .
\end{aligned}
$$

Then $\mathcal{B}(C[0, t])$ coincides with the smallest $\sigma$-algebra generated by $\mathcal{J}$ and there exists a unique probability measure $w_{\varphi}$ on $(C[0, t], \mathcal{B}(C[0, t]))$ such that $w_{\varphi}(I)=m_{\varphi}(I)$ for all $I$ in $\mathcal{J}$. This measure $w_{\varphi}$ is called an analogue of the Wiener measure associated with the probability measure $\varphi[6,9]$. Let $r$ be a positive integer and $C^{r}=C^{r}[0, t]$ be the product space of $C[0, t]$ with the product measure $w_{\varphi}^{r}$. Since $C[0, t]$ is a separable Banach space, we have $\mathcal{B}\left(C^{r}[0, t]\right)=\prod_{j=1}^{r} \mathcal{B}(C[0, t])$. This probability measure space $\left(C^{r}[0, t], \mathcal{B}\left(C^{r}[0, t]\right), w_{\varphi}^{r}\right)$ is called an analogue of the $r$-dimensional Wiener space.

Lemma 1.1 ([6, Lemma 2.1]). If $f: \mathbb{R}^{n+1} \rightarrow \mathbb{C}$ is a Borel measurable function, then we have

$$
\begin{aligned}
& \int_{C} f\left(x\left(t_{0}\right), x\left(t_{1}\right), \cdots, x\left(t_{n}\right)\right) d w_{\varphi}(x) \\
\stackrel{*}{=} & {\left[\prod_{j=1}^{n} \frac{1}{2 \pi\left(t_{j}-t_{j-1}\right)}\right]^{\frac{1}{2}} \int_{\mathbb{R}} \int_{\mathbb{R}^{n}} f\left(u_{0}, u_{1}, \cdots, u_{n}\right) \exp \left\{-\frac{1}{2} \sum_{j=1}^{n} \frac{\left(u_{j}-u_{j-1}\right)^{2}}{t_{j}-t_{j-1}}\right\} } \\
& d\left(u_{1}, \cdots, u_{n}\right) d \varphi\left(u_{0}\right)
\end{aligned}
$$

where $\stackrel{*}{=}$ means that if either side exists, then both sides exist and they are equal.

Definition 1.2. Let $F: C^{r}[0, t] \rightarrow \mathbb{C}$ be $w_{\varphi}^{r}$-integrable and let $Z$ be a random vector on $C^{r}[0, t]$ assuming that the value space of $Z$ is a normed space with the Borel $\sigma$-algebra. Then we have the conditional expectation $E[F \mid Z]$ of $F$ given $Z$ from a well known probability theory. Further, there exists a $P_{Z}$-integrable complex-valued function $\Xi$ on the value space of $Z$ such that $E[F \mid Z](x)=(\Xi \circ Z)(x)$ for $w_{\varphi}^{r}$-a.e. $x \in C^{r}[0, t]$, where $P_{Z}$ is the probability distribution of $Z$. The function $\Xi$ is called the conditional $w_{\varphi}^{r}$-integral of $F$ given $Z$ and it is also denoted by $E[F \mid Z]$.

\section{The simple formulas for conditional $\mathbf{w}_{\varphi}^{\mathbf{r}}$-integrals}

Let $0=t_{0}<t_{1}<\cdots<t_{n-1}<t_{n}=t$ be a partition of $[0, t]$. For any $x$ in $C^{r}[0, t]$, define the polygonal function $[x]$ of $x$ on $[0, t]$ by

$$
[x](s)=\sum_{j=1}^{n} \chi_{\left(t_{j-1}, t_{j}\right]}(s)\left(\frac{t_{j}-s}{t_{j}-t_{j-1}} x\left(t_{j-1}\right)+\frac{s-t_{j-1}}{t_{j}-t_{j-1}} x\left(t_{j}\right)\right)+\chi_{\{0\}}(s) x(0)
$$


for $s \in[0, t]$, where $\chi_{\left(t_{j-1}, t_{j}\right]}$ and $\chi_{\{0\}}$ denote the indicator functions of $\left(t_{j-1}, t_{j}\right]$ and $\{0\}$, respectively. For $\vec{\xi}_{n}=\left(\xi_{0}, \xi_{1}, \cdots, \xi_{n-1}, \xi_{n}\right) \in \mathbb{R}^{(n+1) r}$, we define the polygonal function $\left[\vec{\xi}_{n}\right]$ of $\vec{\xi}_{n}$ as $(2.1)$ replacing $x\left(t_{j}\right)$ by $\xi_{j}$ for $j=0,1, \cdots, n$.

Now, we introduce a lemma which is useful to prove several theorems. The proof follows immediately from Corollary 2.5 in [4].

Lemma 2.1. The processes $\left\{x(s)-[x](s): t_{j-1} \leq s \leq t_{j}\right\}$ on $C^{r}[0, t]$, for $j=$ $1, \cdots, n$, are stochastically independent.

In the following two theorems, we introduce two simple formulas for the conditional $w_{\varphi}^{r}$-integrals on $C^{r}[0, t]$. Their proofs follow immediately from Theorem 2.9 in [4] and Theorem 2.5 in [5].

Theorem 2.2. Let $F: C^{r}[0, t] \rightarrow \mathbb{C}$ be $w_{\varphi}^{r}$-integrable and $X_{t}: C^{r}[0, t] \rightarrow \mathbb{R}^{(n+1) r}$ be given by

$$
X_{t}(x)=\left(x\left(t_{0}\right), x\left(t_{1}\right), \cdots, x\left(t_{n-1}\right), x\left(t_{n}\right)\right)
$$

for $x \in C^{r}[0, t]$. Then we have for $P_{X_{t}}$-a.e. $\vec{\xi}_{n} \in \mathbb{R}^{(n+1) r}$

$$
E\left[F \mid X_{t}\right]\left(\vec{\xi}_{n}\right)=E\left[F\left(x-[x]+\left[\vec{\xi}_{n}\right]\right)\right],
$$

where $P_{X_{t}}$ is the probability distribution of $X_{t}$ on $\left(\mathbb{R}^{(n+1) r}, \mathcal{B}\left(\mathbb{R}^{(n+1) r}\right)\right)$.

Theorem 2.3. Let $F: C^{r}[0, t] \rightarrow \mathbb{C}$ be $w_{\varphi}^{r}$-integrable and $Y_{t}: C^{r}[0, t] \rightarrow \mathbb{R}^{n r}$ be given by

$$
Y_{t}(x)=\left(x\left(t_{0}\right), x\left(t_{1}\right), \cdots, x\left(t_{n-1}\right)\right)
$$

for $x \in C^{r}[0, t]$. Then we have for $P_{Y_{t}}$-a.e. $\vec{\xi}_{n-1}=\left(\xi_{0}, \xi_{1}, \cdots, \xi_{n-1}\right) \in \mathbb{R}^{n r}$

$$
\begin{aligned}
E\left[F \mid Y_{t}\right]\left(\vec{\xi}_{n-1}\right)= & {\left[\frac{1}{2 \pi\left(t-t_{n-1}\right)}\right]^{\frac{r}{2}} \int_{\mathbb{R}^{r}} E\left[F\left(x-[x]+\left[\vec{\xi}_{n}\right]\right)\right] } \\
& \times \exp \left\{-\frac{\left\|\xi_{n}-\xi_{n-1}\right\|_{\mathbb{R}^{r}}^{2}}{2\left(t-t_{n-1}\right)}\right\} d \xi_{n}
\end{aligned}
$$

where $P_{Y_{t}}$ is the probability distribution of $Y_{t}$ on $\left(\mathbb{R}^{n r}, \mathcal{B}\left(\mathbb{R}^{n r}\right)\right)$ and $\left[\vec{\xi}_{n}\right]$ denotes the polygonal function of $\left(\xi_{0}, \xi_{1}, \cdots, \xi_{n-1}, \xi_{n}\right) \in \mathbb{R}^{(n+1) r}$.

For a function $F: C^{r}[0, t] \rightarrow \mathbb{C}$ and $\lambda>0$, let $F^{\lambda}(x)=F\left(\lambda^{-\frac{1}{2}} x\right), X_{t}^{\lambda}(x)=$ $X_{t}\left(\lambda^{-\frac{1}{2}} x\right)$ and $Y_{t}^{\lambda}(x)=Y_{t}\left(\lambda^{-\frac{1}{2}} x\right)$, where $X_{t}$ and $Y_{t}$ are given by (2.2) and (2.4), respectively. Suppose that $E\left[F^{\lambda}\right]$ exists for each $\lambda>0$. By the definition of the conditional $w_{\varphi}^{r}$-integral and (2.3), we have

$$
E\left[F^{\lambda} \mid X_{t}^{\lambda}\right]\left(\vec{\xi}_{n}\right)=E\left[F\left(\lambda^{-\frac{1}{2}}(x-[x])+\left[\vec{\xi}_{n}\right]\right)\right]
$$


for $P_{X_{t}^{\lambda}}$-a.e. $\vec{\xi}_{n} \in \mathbb{R}^{(n+1) r}$, where $P_{X_{t}^{\lambda}}$ is the probability distribution of $X_{t}^{\lambda}$ on the Borel class of $\mathbb{R}^{(n+1) r}$. Throughout this paper, for $y \in C^{r}[0, t]$ let

$$
I_{F}^{\lambda}\left(y, \vec{\xi}_{n}\right)=E\left[F\left(y+\lambda^{-\frac{1}{2}}(x-[x])+\left[\vec{\xi}_{n}\right]\right)\right]
$$

unless otherwise specified, where the expectation is taken over the variable $x$. Moreover, under the notations used in Theorem 2.3, we have by (2.5)

$$
\begin{aligned}
& E\left[F^{\lambda} \mid Y_{t}^{\lambda}\right]\left(\vec{\xi}_{n-1}\right) \\
= & {\left[\frac{\lambda}{2 \pi\left(t-t_{n-1}\right)}\right]^{\frac{r}{2}} \int_{\mathbb{R}^{r}} I_{F}^{\lambda}\left(0, \vec{\xi}_{n}\right) \exp \left\{-\frac{\lambda}{2} \frac{\left\|\xi_{n}-\xi_{n-1}\right\|_{\mathbb{R}^{r}}^{2}}{t-t_{n-1}}\right\} d \xi_{n} }
\end{aligned}
$$

for $P_{Y_{t}^{\lambda}}$-a.e. $\vec{\xi}_{n-1} \in \mathbb{R}^{n r}$, where $P_{Y_{t}^{\lambda}}$ is the probability distribution of $Y_{t}^{\lambda}$ on the Borel class of $\mathbb{R}^{n r}$. From now on, for $y \in C^{r}[0, t]$ let $K_{F}^{\lambda}\left(y, \vec{\xi}_{n-1}\right)$ be given by (2.6) replacing 0 by $y$.

If $I_{F}^{\lambda}\left(0, \vec{\xi}_{n}\right)$ has the analytic extension $J_{\lambda}^{*}(F)\left(\vec{\xi}_{n}\right)$ on $\mathbb{C}_{+}$as a function of $\lambda$, then it is called the conditional analytic Wiener $w_{\varphi}^{r}$-integral of $F$ given $X_{t}$ with the parameter $\lambda$ and denoted by

$$
E^{a n w_{\lambda}}\left[F \mid X_{t}\right]\left(\vec{\xi}_{n}\right)=J_{\lambda}^{*}(F)\left(\vec{\xi}_{n}\right)
$$

for $\vec{\xi}_{n} \in \mathbb{R}^{(n+1) r}$. Moreover, if for a nonzero real $q, E^{a n w_{\lambda}}\left[F \mid X_{t}\right]\left(\vec{\xi}_{n}\right)$ has the limit as $\lambda$ approaches to $-i q$ through $\mathbb{C}_{+}$, then it is called the conditional analytic Feynman $w_{\varphi}^{r}$-integral of $F$ given $X_{t}$ with the parameter $q$ and denoted by

$$
E^{a n f_{q}}\left[F \mid X_{t}\right]\left(\vec{\xi}_{n}\right)=\lim _{\lambda \rightarrow-i q} E^{a n w_{\lambda}}\left[F \mid X_{t}\right]\left(\vec{\xi}_{n}\right) .
$$

Similarly, the definitions of $E^{a n w_{\lambda}}\left[F \mid Y_{t}\right]\left(\vec{\xi}_{n-1}\right)$ and $E^{a n f_{q}}\left[F \mid Y_{t}\right]\left(\vec{\xi}_{n-1}\right)$ are understood with $K_{F}^{\lambda}\left(0, \vec{\xi}_{n-1}\right)$.

\section{Time-dependent conditional Fourier-Feynman transform}

For a given extended real number $p$ with $1<p \leq \infty$, suppose that $p$ and $p^{\prime}$ are related by $\frac{1}{p}+\frac{1}{p^{\prime}}=1$ (possibly $p^{\prime}=1$ if $p=\infty$ ). Let $F_{n}$ and $F$ be measurable functions such that for $\rho>0$

$$
\lim _{n \rightarrow \infty} \int_{C^{r}}\left|F_{n}(\rho y)-F(\rho y)\right|^{p^{\prime}} d w_{\varphi}^{r}(y)=0 .
$$

Then we write

$$
\underset{n \rightarrow \infty}{\lim .}\left(w^{p^{\prime}}\right)\left(F_{n}\right) \approx F
$$

and call $F$ the limit in the mean of order $p^{\prime}$. A similar definition is understood when $n$ is replaced by a continuously varying parameter. 
Now, we define an analytic conditional Fourier-Feynman transform of the functions on $C^{r}[0, t]$.

Definition 3.1. Let $F$ be defined on $C^{r}[0, t]$ and let $X_{t}$ be given by (2.2). For $\lambda \in \mathbb{C}_{+}$and for $w_{\varphi}^{r}$-a.e. $y \in C^{r}[0, t]$, let

$$
T_{\lambda}\left[F \mid X_{t}\right]\left(y, \vec{\xi}_{n}\right)=E^{a n w_{\lambda}}\left[F(y+\cdot) \mid X_{t}\right]\left(\vec{\xi}_{n}\right)
$$

for $P_{X_{t}}$-a.e. $\vec{\xi}_{n} \in \mathbb{R}^{n+1}$ if it exists. For a non-zero real $q$ and for $w_{\varphi}^{r}$-a.e. $y \in C^{r}[0, t]$, we define the $L_{1}$ analytic conditional Fourier-Feynman transform $T_{q}^{(1)}\left[F \mid X_{t}\right]$ of $F$ by the formula

$$
T_{q}^{(1)}\left[F \mid X_{t}\right]\left(y, \vec{\xi}_{n}\right)=E^{a n f_{q}}\left[F(y+\cdot) \mid X_{t}\right]\left(\vec{\xi}_{n}\right)
$$

for $P_{X_{t}}$-a.e. $\vec{\xi}_{n} \in \mathbb{R}^{n+1}$ if it exists. For $1<p \leq \infty$ we define the $L_{p}$ analytic conditional Fourier-Feynman transform $T_{q}^{(p)}\left[F \mid X_{t}\right]$ of $F$ by the formula

$$
T_{q}^{(p)}\left[F \mid X_{t}\right]\left(\cdot, \vec{\xi}_{n}\right) \approx \operatorname{li.i.m.~}_{\lambda \rightarrow-i q}\left(w^{p^{\prime}}\right)\left(T_{\lambda}\left[F \mid X_{t}\right]\left(\cdot, \vec{\xi}_{n}\right)\right)
$$

for $P_{X_{t}}$-a.e. $\vec{\xi}_{n} \in \mathbb{R}^{n+1}$, where $\lambda$ approaches to $-i q$ through $\mathbb{C}_{+}$.

Similar definitions are understood with $K_{F}^{\lambda}\left(y, \vec{\xi}_{n-1}\right)$ if we replace $X_{t}$ by $Y_{t}$ which is given by (2.4).

Let $\eta$ be a complex valued Borel measure on $[0, t]$. Then $\eta=\mu+\nu$ can be decomposed uniquely into the sum of a continuous measure $\mu$ and a discrete measure $\nu$. Further, let $\delta_{p_{l, j}}$ denote the Dirac measure with total mass 1 concentrated at $p_{l, j}$.

Let $\mathcal{M}\left(\mathbb{R}^{r}\right)$ be the class of all complex Borel measures on $\mathbb{R}^{r}$ and $\mathcal{G}^{*}$ be the set of all $\mathbb{C}$-valued functions $\theta$ on $[0, \infty) \times \mathbb{R}^{r}$ which have the form

$$
\theta(s, \vec{u})=\int_{\mathbb{R}^{r}} \exp \{i\langle\vec{u}, \vec{v}\rangle\} d \sigma_{s}(\vec{v})
$$

where $\langle\cdot, \cdot\rangle$ denotes the dot product on $\mathbb{R}^{r}$ and $\left\{\sigma_{s}: s \in[0, \infty)\right\}$ is the family from $\mathcal{M}\left(\mathbb{R}^{r}\right)$ satisfying the following conditions;

(i) for each Borel subset $E$ of $\mathbb{R}^{r}, \sigma_{s}(E)$ is a Borel measurable function of $s$ on $[0, t]$,

(ii) $\left\|\sigma_{s}\right\| \in L_{1}([0, t], \mathcal{B}([0, t]),|\eta|)$.

Now we have the following theorem.

Theorem 3.2. Let $X_{t}$ be given by (2.2) and let $\eta=\mu+\sum_{l=1}^{n} \sum_{j=1}^{r_{l}} w_{l, j} \delta_{p_{l, j}}$, where $w_{l, j} \in \mathbb{C}$ for all $(l, j)$ and $0=t_{0}<p_{1,1}<p_{1,2}<\cdots<p_{1, r_{1}}<t_{1}<p_{2,1}<\cdots<$ 
$p_{2, r_{2}}<t_{2}<\cdots<t_{n-1}<p_{n, 1}<\cdots<p_{n, r_{n}}<t_{n}=t$. Further, let $k$ be a positive integer and let

$$
F_{k}(x)=\left[\int_{0}^{t} \theta(s, x(s)) d \eta(s)\right]^{k} \text { for } x \in C^{r}[0, t]
$$

where $\theta \in \mathcal{G}^{*}$ is given by (3.1). Then for $\lambda \in \mathbb{C}_{+}, y \in C^{r}[0, t]$ and $\vec{\xi}_{n}=$ $\left(\xi_{0}, \xi_{1}, \cdots, \xi_{n}\right) \in \mathbb{R}^{(n+1) r}, T_{\lambda}\left[F_{k} \mid X_{t}\right]\left(y, \vec{\xi}_{n}\right)$ exists and it is given by

$$
T_{\lambda}\left[F_{k} \mid X_{t}\right]\left(y, \vec{\xi}_{n}\right)=k ! \sum_{q_{1}+\cdots+q_{n}=k} \prod_{l=1}^{n} A\left(l, \lambda, y, \xi_{l-1}, \xi_{l}, q_{l}\right)
$$

where

$$
\begin{aligned}
& A\left(l, \lambda, y, \xi_{l-1}, \xi_{l}, q_{l}\right) \\
& =\sum_{m_{l, 0}+m_{l, 1}+\cdots+m_{l, r_{l}}=q_{l}}\left(\prod_{j=1}^{r_{l}} \frac{w_{l, j}^{m_{l, j}}}{m_{l, j} !} \sum_{j_{0}+j_{1}+\cdots+j_{r_{l}}=m_{l, 0}} \int_{\Delta_{m_{l, 0} ; j_{0}, \cdots, j_{r_{l}}}} \int_{\mathbb{R}^{q_{l} r}} D(l, y,\right. \\
& \left.\xi_{l-1}, \xi_{l}, \vec{v}_{l}, \vec{h}_{l}, \vec{s}_{l}\right) \exp \left\{-\frac{1}{2 \lambda} \sum_{u=0}^{r_{l}} \sum_{v=1}^{j_{u}+1}\left(s_{l, u, v}-s_{l, u, v-1}\right) \| \sum_{\beta=u+1}^{r_{l}} \sum_{\gamma=1}^{j_{\beta}+1} \frac{t_{l}-s_{l, \beta, \gamma}}{t_{l}-t_{l-1}}\right. \\
& \times \vec{v}_{l, \beta, \gamma}+\sum_{\gamma=v}^{j_{u}+1} \frac{t_{l}-s_{l, u, \gamma}}{t_{l}-t_{l-1}} \vec{v}_{l, u, \gamma}+\sum_{\gamma=1}^{v-1} \frac{t_{l-1}-s_{l, u, \gamma}}{t_{l}-t_{l-1}} \vec{v}_{l, u, \gamma}+\sum_{\beta=0}^{u-1} \sum_{\gamma=1}^{j_{\beta}+1} \frac{t_{l-1}-s_{l, \beta, \gamma}}{t_{l}-t_{l-1}} \\
& \left.\times \vec{v}_{l, \beta, \gamma} \|_{\mathbb{R}^{r}}^{2}\right\} d\left(\prod_{u=0}^{r_{l}} \prod_{v=1}^{j_{u}} \sigma_{s_{l, u, v}} \times \prod_{u=1}^{r_{l}} \sigma_{p_{l, u}}^{m_{l, u}}\right)\left(\vec{v}_{l}, \vec{h}_{l}\right) d \mu^{m_{l, 0}}\left(\vec{s}_{l}\right)
\end{aligned}
$$

with the conventions those $s_{l, 0,0}=t_{l-1}, s_{l, u, 0}=p_{l, u}=s_{l, u-1, j_{u-1}+1}$ for $u=1, \cdots, r_{l}, \quad s_{l, r_{l}, j_{r_{l}}+1}=t_{l}, \quad \vec{s}_{l}=\left(s_{l, 0,1}, \cdots, s_{l, 0, j_{0}}, \cdots, s_{l, r_{l}, 1}, \cdots, s_{l, r_{l}, j_{r_{l}}}\right)$, $\Delta_{m_{l, 0} ; j_{0}, \cdots, j_{r_{l}}}=\left\{\vec{s}_{l}: t_{l-1}<s_{l, 0,1}<\cdots<s_{l, 0, j_{0}}<p_{l, 1}<s_{l, 1,1}<\cdots<s_{l, 1, j_{1}}<\right.$ $\left.p_{l, 2}<\cdots<p_{l, r_{l}}<s_{l, r_{l}, 1}<\cdots<s_{l, r_{l}, j_{r_{l}}}<t_{l}\right\}, \vec{v}_{l}=\left(\vec{v}_{l, 0,1}, \cdots, \vec{v}_{l, 0, j_{0}}, \vec{v}_{l, 1,1}\right.$, $\left.\cdots, \vec{v}_{l, 1, j_{1}}, \cdots, \vec{v}_{l, r_{l}, 1}, \cdots, \vec{v}_{l, r_{l}, j_{r_{l}}}\right), \vec{v}_{l, r_{l}, j_{r_{l}}+1}=\overrightarrow{0} \in \mathbb{R}^{r}, \vec{h}_{l}=\left(\vec{h}_{l, 1,1}, \cdots\right.$, $\left.\vec{h}_{l, 1, m_{l, 1}}, \vec{h}_{l, 2,1}, \cdots, \vec{h}_{l, 2, m_{l, 2}}, \cdots, \vec{h}_{l, r_{l}, 1}, \cdots, \vec{h}_{l, r_{l}, m_{l, r_{l}}}\right) ; \vec{v}_{l, u-1, j_{u-1}+1}=\sum_{v=1}^{m_{l, u}} \vec{h}_{l, u, v}$ for $u=1, \cdots, r_{l}$,

$$
\begin{aligned}
& D\left(l, y, \xi_{l-1}, \xi_{l}, \vec{v}_{l}, \vec{h}_{l}, \vec{s}_{l}\right) \\
= & \exp \left\{i \sum_{u=0}^{r_{l}} \sum_{v=1}^{j_{u}+1}\left\langle y\left(s_{l, u, v}\right)+\frac{t_{l}-s_{l, u, v}}{t_{l}-t_{l-1}} \xi_{l-1}+\frac{s_{l, u, v}-t_{l-1}}{t_{l}-t_{l-1}} \xi_{l}, \vec{v}_{l, u, v}\right\rangle\right\},
\end{aligned}
$$

$\sum_{\beta=u+1}^{r_{l}} \sum_{\gamma=1}^{j_{\beta}+1} \frac{t_{l}-s_{l, \beta, \gamma}}{t_{l}-t_{l-1}} \vec{v}_{l, \beta, \gamma}=\overrightarrow{0}$ if $u=r_{l}, \sum_{\gamma=1}^{v-1} \frac{t_{l-1}-s_{l, u, \gamma}}{t_{l}-t_{l-1}} \vec{v}_{l, u, \gamma}=\overrightarrow{0}$ if $v=1$ and $\sum_{\beta=0}^{u-1} \sum_{\gamma=1}^{j_{\beta}+1} \frac{t_{l-1}-s_{l, \beta, \gamma}}{t_{l}-t_{l-1}} \vec{v}_{l, \beta, \gamma}=\overrightarrow{0}$ if $u=0$. 
Proof. For $\lambda>0, y \in C^{r}[0, t]$ and $\vec{\xi}_{n}=\left(\xi_{0}, \xi_{1}, \cdots, \xi_{n}\right) \in \mathbb{R}^{(n+1) r}$, we have by Lemma 2.1 and the binomial expansion

$$
\begin{aligned}
& I_{F_{k}}^{\lambda}\left(y, \vec{\xi}_{n}\right) \\
= & \sum_{q_{1}+\cdots+q_{n}=k} \frac{k !}{q_{1} ! \cdots q_{n} !} \prod_{l=1}^{n} \int_{C^{r}}\left[\int_{t_{l-1}}^{t_{l}} \theta\left(s, y(s)+\lambda^{-\frac{1}{2}}(x(s)-[x](s))+\left[\vec{\xi}_{n}\right](s)\right)\right. \\
& \left.d \mu(s)+\sum_{j=1}^{r_{l}} w_{l, j} \theta\left(p_{l, j}, y\left(p_{l, j}\right)+\lambda^{-\frac{1}{2}}\left(x\left(p_{l, j}\right)-[x]\left(p_{l, j}\right)\right)+\left[\vec{\xi}_{n}\right]\left(p_{l, j}\right)\right)\right]^{q_{l}} d w_{\varphi}^{r}(x) .
\end{aligned}
$$

Using the simplex method [7] and the Fubini's theorem we have by (3.1)

$$
\begin{aligned}
& I_{F_{k}}^{\lambda}\left(y, \vec{\xi}_{n}\right) \\
= & k ! \sum_{q_{1}+\cdots+q_{n}=k} \prod_{l=1}^{n} \sum_{m_{l, 0}+m_{l, 1}+\cdots+m_{l, r_{l}}=q_{l}}\left(\prod_{j=1}^{r_{l}} \frac{w_{l, j}^{m_{l, j}}}{m_{l, j} !}\right) \sum_{j_{0}+j_{1}+\cdots+j_{r_{l}}=m_{l, 0}} \\
& \int_{\Delta_{m_{l, 0} ; j_{0}, \cdots, j_{l}}} \int_{C^{r}}\left[\int _ { \mathbb { R } ^ { m _ { l , 0 } r } } \operatorname { e x p } \left\{i \sum _ { u = 0 } ^ { r _ { l } } \sum _ { v = 1 } ^ { j _ { u } } \left\langley\left(s_{l, u, v}\right)+\lambda^{-\frac{1}{2}}\left(x\left(s_{l, u, v}\right)-\right.\right.\right.\right. \\
& {\left.\left.\left.\left.[x]\left(s_{l, u, v}\right)\right)+\left[\vec{\xi}_{n}\right]\left(s_{l, u, v}\right), \vec{v}_{l, u, v}\right\rangle\right\} d\left(\prod_{u=0}^{r_{l}} \prod_{v=1}^{j_{u}} \sigma_{s_{l, u, v}}\right)\left(\vec{v}_{l}\right)\right]\left[\int_{\mathbb{R}^{\left(m_{l, 1}+\cdots+m_{l, r_{l}}\right) r}}\right.} \\
& \exp \left\{i \sum_{u=1}^{r_{l}} \sum_{v=1}^{m_{l, u}}\left\langle y\left(s_{l, u, 0}\right)+\lambda^{-\frac{1}{2}}\left(x\left(s_{l, u, 0}\right)-[x]\left(s_{l, u, 0}\right)\right)+\left[\vec{\xi}_{n}\right]\left(s_{l, u, 0}\right), \vec{h}_{l, u, v}\right\rangle\right\} \\
& \left.d\left(\prod_{u=1}^{r_{l}} \sigma_{p_{l, u}}^{m_{l, u}}\right)\left(\vec{h}_{l}\right)\right] d w_{\varphi}^{r}(x) d \mu^{m_{l, 0}}\left(\vec{s}_{l}\right),
\end{aligned}
$$

where $\vec{s}_{l}, \vec{v}_{l}, \vec{h}_{l}$ and $\Delta_{m_{l, 0} ; j_{0}, \cdots, j_{r_{l}}}$ are given by the assumptions, and $s_{l, u, 0}=p_{l, u}$ for $u=1, \cdots, r_{l}$. For $u=1, \cdots, r_{l}$, let $s_{l, u-1, j_{u-1}+1}=s_{l, u, 0}, \vec{v}_{l, u-1, j_{u-1}+1}=$ $\sum_{v=1}^{m_{l, u}} \vec{h}_{l, u, v}, s_{l, r_{l}, j_{r_{l}}+1}=t_{l}, s_{l, 0,0}=t_{l-1}$ and $\vec{v}_{l, r_{l}, j_{r_{l}}+1}=\overrightarrow{0} \in \mathbb{R}^{r}$. Then we have

$$
\begin{aligned}
& I_{F_{k}}^{\lambda}\left(y, \vec{\xi}_{n}\right) \\
= & k ! \sum_{q_{1}+\cdots+q_{n}=k} \prod_{l=1}^{n} \sum_{m_{l, 0}+m_{l, 1}+\cdots+m_{l, r_{l}}=q_{l}}\left(\prod_{j=1}^{r_{l}} \frac{w_{l, j}^{m_{l, j}}}{m_{l, j} !}\right)_{j_{0}+j_{1}+\cdots+j_{r_{l}}=m_{l, 0}} \sum_{\Delta_{m_{l, 0} ; j_{0}, \cdots, j_{r_{l}}}} \int_{\mathbb{R}_{q_{l} r}} D\left(l, y, \xi_{l-1}, \xi_{l}, \vec{v}_{l}, \vec{h}_{l}, \vec{s}_{l}\right) \int_{C^{r}} \exp \left\{i \lambda^{-\frac{1}{2}} \sum_{u=0}^{r_{l}} \sum_{v=1}^{j_{u}+1}\right. \\
& \left\langle\frac{t_{l}-s_{l, u, v}}{t_{l}-t_{l-1}}\left(x\left(s_{l, u, v}\right)-x\left(t_{l-1}\right)\right)+\frac{t_{l-1}-s_{l, u, v}}{t_{l}-t_{l-1}}\left(x\left(t_{l}\right)-x\left(s_{l, u, v}\right)\right),\right. \\
& \left.\left.\vec{v}_{l, u, v}\right\rangle\right\} d w_{\varphi}^{r}(x) d\left(\prod_{u=0}^{r_{l}} \prod_{v=1}^{j_{u}} \sigma_{s_{l, u, v}} \times \prod_{u=1}^{r_{l}} \sigma_{p_{l, u}}^{m_{l, u}}\right)\left(\vec{v}_{l}, \vec{h}_{l}\right) d \mu^{m_{l, 0}\left(\vec{s}_{l}\right)}
\end{aligned}
$$


where $D\left(l, y, \xi_{l-1}, \xi_{l}, \vec{v}_{l}, \vec{h}_{l}, \vec{s}_{l}\right)$ is given by (3.3). Let

$$
S(1)=\left[\prod_{u=0}^{r_{1}} \prod_{v=1}^{j_{u}+1} \frac{1}{2 \pi\left(s_{1, u, v}-s_{1, u, v-1}\right)}\right]^{\frac{r}{2}}
$$

and for $l=2, \cdots, n$ let

$$
S(l)=\left[\frac{1}{2 \pi t_{l-1}} \prod_{u=0}^{r_{l}} \prod_{v=1}^{j_{u}+1} \frac{1}{2 \pi\left(s_{l, u, v}-s_{l, u, v-1}\right)}\right]^{\frac{r}{2}} .
$$

Let $m(1)=m_{1,0}+r_{1}+1$ and $m(l)=m_{l, 0}+r_{l}+2$ if $l=2, \cdots, n$. Then we have by an application of Lemma 1.1

$$
\begin{aligned}
& I_{F_{k}}^{\lambda}\left(y, \vec{\xi}_{n}\right) \\
= & k ! \sum_{q_{1}+\cdots+q_{n}=k} \prod_{l=1}^{n} \sum_{m_{l, 0}+m_{l, 1}+\cdots+m_{l, r_{l}}=q_{l}}\left(\prod_{j=1}^{r_{l}} \frac{w_{l, j}^{m_{l, j}}}{m_{l, j} !}\right)_{j_{0}+j_{1}+\cdots+j_{r_{l}}=m_{l, 0}} \sum_{\Delta_{m_{l, 0} ; j_{0}, \cdots, j_{r_{l}}}} \int_{\mathbb{R}^{q_{l} r}} D\left(l, y, \xi_{l-1}, \xi_{l}, \vec{v}_{l}, \vec{h}_{l}, \vec{s}_{l}\right) S(l) \int_{\mathbb{R}^{r}} \int_{\mathbb{R}^{m(l) r}} \exp \left\{i \lambda^{-\frac{1}{2}} \sum_{u=0}^{r_{l}} \sum_{v=1}^{j_{u}+1}\right. \\
& \left\langle\frac{t_{l}-s_{l, u, v}}{t_{l}-t_{l-1}}\left(\vec{\zeta}_{l, u, v}-\vec{\zeta}_{l, 0,0}\right)+\frac{t_{l-1}-s_{l, u, v}}{t_{l}-t_{l-1}}\left(\vec{\zeta}_{l, r_{l}, j_{r_{l}}+1}-\vec{\zeta}_{l, u, v}\right), \vec{v}_{l, u, v}\right\rangle-\frac{1}{2} \sum_{u=0}^{r_{l}} \\
& \left.\sum_{v=1}^{j_{u}+1} \frac{\left\|\vec{\zeta}_{l, u, v}-\vec{\zeta}_{l, u, v-1}\right\|_{\mathbb{R}^{r}}^{2}}{s_{l, u, v}-s_{l, u, v-1}}-\frac{\left\|\vec{\zeta}_{l, 0,0}-\vec{\zeta}_{1,0,0}\right\|_{\mathbb{R}^{r}}^{2}}{2 t_{l-1}+\delta_{l 1}}\right\} d \vec{\zeta}_{l} d \varphi^{r}\left(\vec{\zeta}_{1,0,0}\right) d\left(\prod_{u=0}^{r_{l}} \prod_{v=1}^{j_{u}} \sigma_{s_{l, u, v}}\right. \\
& \left.\times \prod_{u=1}^{r_{l}} \sigma_{p_{l, u}}^{m_{l, u}}\right)\left(\vec{v}_{l}, \vec{h}_{l}\right) d \mu^{m_{l, 0}}\left(\vec{s}_{l}\right)
\end{aligned}
$$

where $\vec{\zeta}_{l, u-1, j_{u-1}+1}=\vec{\zeta}_{l, u, 0}$ for $u=1, \cdots, r_{l}$ and $\vec{\zeta}_{1}=\left(\vec{\zeta}_{1,0,1}, \cdots, \vec{\zeta}_{1,0, j_{0}+1}, \vec{\zeta}_{1,1,1}\right.$, $\left.\cdots, \vec{\zeta}_{1,1, j_{1}+1}, \cdots, \vec{\zeta}_{1, r_{1}, 1}, \cdots, \vec{\zeta}_{1, r_{1}, j_{r_{1}}+1}\right), \vec{\zeta}_{l}=\left(\vec{\zeta}_{l, 0,0}, \vec{\zeta}_{l, 0,1}, \cdots, \vec{\zeta}_{l, 0, j_{0}+1}, \vec{\zeta}_{l, 1,1}, \cdots\right.$, $\left.\vec{\zeta}_{l, 1, j_{1}+1}, \cdots, \vec{\zeta}_{l, r_{l}, 1}, \cdots, \vec{\zeta}_{l, r_{l}, j_{r_{l}}+1}\right)$ if $l=2, \cdots, n$. Let $\vec{\eta}_{l, u, v}=\vec{\zeta}_{l, u, v}-\vec{\zeta}_{l, u, v-1}$ for $u=0,1, \cdots, r_{l} ; v=1, \cdots, j_{u}+1$ and $\vec{\eta}_{1,0,0}=\overrightarrow{0}, \vec{\eta}_{l, 0,0}=\vec{\zeta}_{l, 0,0}-\vec{\zeta}_{1,0,0}$ if $l=2, \cdots, n$. Then we have by the change of variable theorem

$$
\begin{aligned}
& I_{F_{k}}^{\lambda}\left(y, \vec{\xi}_{n}\right) \\
= & k ! \sum_{q_{1}+\cdots+q_{n}=k} \prod_{l=1}^{n} \sum_{m_{l, 0}+m_{l, 1}+\cdots+m_{l, r_{l}}=q_{l}}\left(\prod_{j=1}^{r_{l}} \frac{w_{l, j}^{m_{l, j}}}{m_{l, j} !}\right)_{j_{0}+j_{1}+\cdots+j_{r_{l}}=m_{l, 0}} \sum \int_{\Delta_{m_{l, 0} ; j_{0}, \cdots, j_{r_{l}}}} \int_{\mathbb{R}^{q_{l} r}} D\left(l, y, \xi_{l-1}, \xi_{l}, \vec{v}_{l}, \vec{h}_{l}, \vec{s}_{l}\right) S(l) \int_{\mathbb{R}^{m(l) r}} \exp \left\{i \lambda^{-\frac{1}{2}} \sum_{u=0}^{r_{l}} \sum_{v=1}^{j_{u}+1}\right.
\end{aligned}
$$




$$
\begin{aligned}
& \left\langle\frac{t_{l}-s_{l, u, v}}{t_{l}-t_{l-1}}\left(\sum_{\beta=0}^{u-1} \sum_{\gamma=1}^{j_{\beta}+1} \vec{\eta}_{l, \beta, \gamma}+\sum_{\gamma=1}^{v} \vec{\eta}_{l, u, \gamma}\right)+\frac{t_{l-1}-s_{l, u, v}}{t_{l}-t_{l-1}}\left(\sum_{\gamma=v+1}^{j_{u}+1} \vec{\eta}_{l, u, \gamma}+\right.\right. \\
& \left.\left.\left.\sum_{\beta=u+1}^{r_{l}} \sum_{\gamma=1}^{j_{\beta}+1} \vec{\eta}_{l, \beta, \gamma}\right), \vec{v}_{l, u, v}\right\rangle-\frac{1}{2} \sum_{u=0}^{r_{l}} \sum_{v=1}^{j_{u}+1} \frac{\left\|\vec{\eta}_{l, u, v}\right\|_{\mathbb{R}^{r}}^{2}}{s_{l, u, v}-s_{l, u, v-1}}-\frac{\left\|\vec{\eta}_{l, 0,0}\right\|_{\mathbb{R}^{r}}^{2}}{2 t_{l-1}+\delta_{l 1}}\right\} \\
& d \vec{\eta}_{l} d\left(\prod_{u=0}^{r_{l}} \prod_{v=1}^{j_{u}} \sigma_{s_{l, u, v}} \times \prod_{u=1}^{r_{l}} \sigma_{p_{l, u}}^{m_{l, u}}\right)\left(\vec{v}_{l}, \vec{h}_{l}\right) d \mu^{m_{l, 0}\left(\vec{s}_{l}\right)}
\end{aligned}
$$

where $\vec{\eta}_{1}=\left(\vec{\eta}_{1,0,1}, \cdots, \vec{\eta}_{1,0, j_{0}+1}, \vec{\eta}_{1,1,1}, \cdots, \vec{\eta}_{1,1, j_{1}+1}, \cdots, \vec{\eta}_{1, r_{1}, 1}, \cdots, \vec{\eta}_{1, r_{1}, j_{r_{1}}+1}\right)$, $\vec{\eta}_{l}=\left(\vec{\eta}_{l, 0,0}, \vec{\eta}_{l, 0,1}, \cdots, \vec{\eta}_{l, 0, j_{0}+1}, \vec{\eta}_{l, 1,1}, \cdots, \vec{\eta}_{l, 1, j_{1}+1}, \cdots, \vec{\eta}_{l, r_{l}, 1}, \cdots, \vec{\eta}_{l, r_{l}, j_{r_{l}}+1}\right)$ if $l \in$ $\{2, \cdots, n\}, \sum_{\beta=0}^{u-1} \sum_{\gamma=1}^{j_{\beta}+1} \vec{\eta}_{l, \beta, \gamma}=\overrightarrow{0}$ if $u=0, \sum_{\gamma=v+1}^{j_{u}+1} \vec{\eta}_{l, u, \gamma}=\overrightarrow{0}$ if $v=j_{u}+1$ and $\sum_{\beta=u+1}^{r_{l}} \sum_{\gamma=1}^{j_{\beta}+1} \vec{\eta}_{l, \beta, \gamma}=\overrightarrow{0}$ if $u=r_{l}$. Now we have

$$
\begin{aligned}
& I_{F_{k}}^{\lambda}\left(y, \vec{\xi}_{n}\right) \\
& =k ! \sum_{q_{1}+\cdots+q_{n}=k} \prod_{l=1}^{n} \sum_{m_{l, 0}+m_{l, 1}+\cdots+m_{l, r_{l}}=q_{l}}\left(\prod_{j=1}^{r_{l}} \frac{w_{l, j}^{m_{l, j}}}{m_{l, j} !}\right)_{j_{0}+j_{1}+\cdots+j_{r_{l}}=m_{l, 0}} \\
& \int_{\Delta_{m_{l, 0} ; j_{0}, \cdots, j_{r_{l}}}} \int_{\mathbb{R}^{q_{l} r}} D\left(l, y, \xi_{l-1}, \xi_{l}, \vec{v}_{l}, \vec{h}_{l}, \vec{s}_{l}\right) S(l) \int_{\mathbb{R}^{m(l) r}} \exp \left\{i \lambda^{-\frac{1}{2}} \sum_{u=0}^{r_{l}} \sum_{v=1}^{j_{u}+1}\langle\right. \\
& \vec{\eta}_{l, u, v}, \sum_{\beta=u+1}^{r_{l}} \sum_{\gamma=1}^{j_{\beta}+1} \frac{t_{l}-s_{l, \beta, \gamma}}{t_{l}-t_{l-1}} \vec{v}_{l, \beta, \gamma}+\sum_{\gamma=v}^{j_{u}+1} \frac{t_{l}-s_{l, u, \gamma}}{t_{l}-t_{l-1}} \vec{v}_{l, u, \gamma}+\sum_{\gamma=1}^{v-1} \frac{t_{l-1}-s_{l, u, \gamma}}{t_{l}-t_{l-1}} \\
& \left.\vec{v}_{l, u, \gamma}+\sum_{\beta=0}^{u-1} \sum_{\gamma=1}^{j_{\beta}+1} \frac{t_{l-1}-s_{l, \beta, \gamma}}{t_{l}-t_{l-1}} \vec{v}_{l, \beta, \gamma}\right\rangle-\frac{1}{2} \sum_{u=0}^{r_{l}} \sum_{v=1}^{j_{u}+1} \frac{\left\|\vec{\eta}_{l, u, v}\right\|_{\mathbb{R}^{r}}^{2}}{s_{l, u, v}-s_{l, u, v-1}}- \\
& \left.\frac{\left\|\vec{\eta}_{l, 0,0}\right\|_{\mathbb{R}^{r}}^{2}}{2 t_{l-1}+\delta_{l 1}}\right\} d \vec{\eta}_{l} d\left(\prod_{u=0}^{r_{l}} \prod_{v=1}^{j_{u}} \sigma_{s_{l, u, v}} \times \prod_{u=1}^{r_{l}} \sigma_{p_{l, u}}^{m_{l, u}}\right)\left(\vec{v}_{l}, \vec{h}_{l}\right) d \mu^{m_{l, 0}}\left(\vec{s}_{l}\right) \\
& =k ! \sum_{q_{1}+\cdots+q_{n}=k} \prod_{l=1}^{n} A\left(l, \lambda, y, \xi_{l-1}, \xi_{l}, q_{l}\right)
\end{aligned}
$$

where the last equality follows from the well known integration formula

$$
\int_{\mathbb{R}} \exp \left\{-a u^{2}+i b u\right\} d u=\left(\frac{\pi}{a}\right)^{\frac{1}{2}} \exp \left\{-\frac{b^{2}}{4 a}\right\}
$$

for $a \in \mathbb{C}_{+}$and any real $b$. By the Morera's theorem, the theorem follows.

Corollary 3.3. Under the assumptions given as in Theorem 3.2, with one exception $\eta=\mu$, that is, assuming that $\eta$ has no discrete part, we have for $\lambda \in \mathbb{C}_{+}, y \in C^{r}[0, t]$ 
and $\vec{\xi}_{n} \in \mathbb{R}^{(n+1) r}$

$$
T_{\lambda}\left[F_{k} \mid X_{t}\right]\left(y, \vec{\xi}_{n}\right)=k ! \sum_{q_{1}+\cdots+q_{n}=k} \prod_{l=1}^{n} A_{\mu}\left(l, \lambda, y, \vec{\xi}_{n}, q_{l}\right)
$$

where

$$
\begin{aligned}
& A_{\mu}\left(l, \lambda, y, \vec{\xi}_{n}, q_{l}\right) \\
= & \int_{\Delta_{q_{l}}} \int_{\mathbb{R}_{q_{l} r}} \exp \left\{i \sum_{u=1}^{q_{l}}\left\langle y\left(s_{l, u}\right)+\left[\vec{\xi}_{n}\right]\left(s_{l, u}\right), \vec{v}_{l, u}\right\rangle-\frac{1}{2 \lambda} \sum_{u=1}^{q_{l}+1}\left(s_{l, u}-s_{l, u-1}\right)\right. \\
& \left.\times\left\|\sum_{\beta=u}^{q_{l}} \frac{t_{l}-s_{l, \beta}}{t_{l}-t_{l-1}} \vec{v}_{l, \beta}+\sum_{\beta=1}^{u-1} \frac{t_{l-1}-s_{l, \beta}}{t_{l}-t_{l-1}} \vec{v}_{l, \beta}\right\|_{\mathbb{R}^{r}}^{2}\right\} d\left(\prod_{u=1}^{q_{l}} \sigma_{s_{l, u}}\right)\left(\vec{v}_{l}\right) d \mu^{q_{l}}\left(\vec{s}_{l}\right)
\end{aligned}
$$

with the conventions those $s_{l, 0}=t_{l-1}, s_{l, q_{l}+1}=t_{l}, \vec{s}_{l}=\left(s_{l, 1}, s_{l, 2}, \cdots, s_{l, q_{l}}\right)$, $\Delta_{q_{l}}=\left\{\vec{s}_{l}: t_{l-1}<s_{l, 1}<s_{l, 2}<\cdots<s_{l, q_{l}}<t_{l}\right\}, \vec{v}_{l}=\left(\vec{v}_{l, 1}, \vec{v}_{l, 2}, \cdots, \vec{v}_{l, q_{l}}\right)$, $\sum_{\beta=u}^{q_{l}} \frac{t_{l}-s_{l, \beta}}{t_{l}-t_{l-1}} \vec{v}_{l, \beta}=\overrightarrow{0}$ if $u=q_{l}+1$ and $\sum_{\beta=1}^{u-1} \frac{t_{l-1}-s_{l, \beta}}{t_{l}-t_{l-1}} \vec{v}_{l, \beta}=\overrightarrow{0}$ if $u=1$.

Corollary 3.4. Under the assumptions given as in Theorem 3.2 with one exception $\eta=\sum_{l=1}^{n} \sum_{j=1}^{r_{l}} w_{l, j} \delta_{p_{l, j}}$, that is, assuming that $\eta$ has no continuous part, we have for $\lambda \in \mathbb{C}_{+}, y \in C^{r}[0, t]$ and $\vec{\xi}_{n} \in \mathbb{R}^{(n+1) r}$

$$
T_{\lambda}\left[F_{k} \mid X_{t}\right]\left(y, \vec{\xi}_{n}\right)=k ! \sum_{q_{1}+\cdots+q_{n}=k} \prod_{l=1}^{n} A_{\nu}\left(l, \lambda, y, \vec{\xi}_{n}, q_{l}\right)
$$

where

$$
\begin{aligned}
& A_{\nu}\left(l, \lambda, y, \vec{\xi}_{n}, q_{l}\right) \\
= & \sum_{m_{l, 1}+\cdots+m_{l, r_{l}}=q_{l}}\left(\prod_{j=1}^{r_{l}} \frac{w_{l, j}^{m_{l, j}}}{m_{l, j} !}\right) \int_{\mathbb{R}_{q_{l} r}} \exp \left\{i \sum_{u=1}^{r_{l}} \sum_{v=1}^{m_{l, u}}\left\langle y\left(p_{l, u}\right)+\left[\vec{\xi}_{n}\right]\left(p_{l, u}\right), \vec{h}_{l, u, v}\right\rangle\right. \\
& -\frac{1}{2 \lambda} \sum_{u=1}^{r_{l}+1}\left(p_{l, u}-p_{l, u-1}\right)\left\|\sum_{\beta=u}^{r_{l}} \sum_{\gamma=1}^{m_{l, \beta}} \frac{t_{l}-p_{l, \beta}}{t_{l}-t_{l-1}} \vec{h}_{l, \beta, \gamma}+\sum_{\beta=1}^{u-1} \sum_{\gamma=1}^{m_{l, \beta}} \frac{t_{l-1}-p_{l, \beta}}{t_{l}-t_{l-1}} \vec{h}_{l, \beta, \gamma}\right\|_{\mathbb{R}^{r}}^{2} \\
& \} d\left(\prod_{u=1}^{r_{l}} \sigma_{p_{l, u}}^{m_{l, u}}\right)\left(\vec{h}_{l}\right)
\end{aligned}
$$

with the conventions those $p_{l, 0}=t_{l-1}, p_{l, r_{l}+1}=t_{l}, \vec{h}_{l}=\left(\vec{h}_{l, 1,1}, \cdots, \vec{h}_{l, 1, m_{l, 1}}, \vec{h}_{l, 2,1}\right.$, $\left.\cdots, \vec{h}_{l, 2, m_{l, 2}}, \cdots, \vec{h}_{l, r_{l}, 1}, \cdots, \vec{h}_{l, r_{l}, m_{l, r_{l}}}\right), \sum_{\beta=u}^{r_{l}} \sum_{\gamma=1}^{m_{l, \beta}} \frac{t_{l}-p_{l, \beta}}{t_{l}-t_{l}-1} \vec{h}_{l, \beta, \gamma}=\overrightarrow{0}$ if $u=r_{l}+1$ and $\sum_{\beta=1}^{u-1} \sum_{\gamma=1}^{m_{l, \beta}} \frac{t_{l-1}-p_{l, \beta}}{t_{l}-t_{l-1}} \vec{h}_{l, \beta, \gamma}=\overrightarrow{0}$ if $u=1$.

Theorem 3.5. Let $1 \leq p \leq \infty$ and $q$ be a nonzero real number. Then, under the 
assumptions given as in Theorem 3.2, $T_{q}^{(p)}\left[F_{k} \mid X_{t}\right]\left(y, \vec{\xi}_{n}\right)$ exists for $y \in C^{r}[0, t]$ and $\vec{\xi}_{n}=\left(\xi_{0}, \xi_{1}, \cdots, \xi_{n}\right) \in \mathbb{R}^{(n+1) r}$, and it is given by

$$
T_{q}^{(p)}\left[F_{k} \mid X_{t}\right]\left(y, \vec{\xi}_{n}\right)=k ! \sum_{q_{1}+\cdots+q_{n}=k} \prod_{l=1}^{n} A\left(l,-i q, y, \xi_{l-1}, \xi_{l}, q_{l}\right)
$$

where $A$ is given as in Theorem 3.2.

Proof. For $1 \leq p \leq \infty$ let $T_{q}^{(p)}\left[F_{k} \mid X_{t}\right]\left(y, \vec{\xi}_{n}\right)$ be given by the right hand side of (3.5). If $\lambda \in \mathbb{C}_{+}$or $\lambda=-i q$, we have by the simplex method and the binomial expansion

$$
\begin{aligned}
& \left|k ! \sum_{q_{1}+\cdots+q_{n}=k} \prod_{l=1}^{n} A\left(l, \lambda, y, \xi_{l-1}, \xi_{l}, q_{l}\right)\right| \\
\leq & k ! \sum_{q_{1}+\cdots+q_{n}=k} \prod_{l=1}^{n}\left[\sum_{m_{l, 0}+m_{l, 1}+\cdots+m_{l, r_{l}}=q_{l}} \frac{1}{m_{l, 0} !}\left[\int_{t_{l-1}}^{t_{l}}\left\|\sigma_{s_{l}}\right\| d|\mu|\left(s_{l}\right)\right]^{m_{l, 0}}\right. \\
& \left.\times \prod_{j=1}^{r_{l}} \frac{\left(\left|w_{l, j}\right|\left\|\sigma_{p_{l, j}}\right\|\right)^{m_{l, j}}}{m_{l, j} !}\right] \\
= & k ! \sum_{q_{1}+\cdots+q_{n}=k} \prod_{l=1}^{n} \frac{1}{q_{l} !}\left[\int_{t_{l-1}}^{t_{l}}\left\|\sigma_{s_{l}}\right\| d|\eta|\left(s_{l}\right)\right]^{q_{l}}=\left[\int_{0}^{t}\left\|\sigma_{s}\right\| d|\eta|(s)\right]^{k}
\end{aligned}
$$

so that for $\lambda \in \mathbb{C}_{+}$we have

$$
\left|T_{\lambda}\left[F_{k} \mid X_{t}\right]\left(y, \vec{\xi}_{n}\right)-T_{q}^{(p)}\left[F_{k} \mid X_{t}\right]\left(y, \vec{\xi}_{n}\right)\right| \leq 2\left[\int_{0}^{t}\left\|\sigma_{s}\right\| d|\eta|(s)\right]^{k} .
$$

Hence when $1<p \leq \infty$, for $\frac{1}{p}+\frac{1}{p^{\prime}}=1$ and $\rho>0$, we have

$$
\begin{aligned}
& \int_{C^{r}}\left|T_{\lambda}\left[F_{k} \mid X_{t}\right]\left(\rho y, \vec{\xi}_{n}\right)-T_{q}^{(p)}\left[F_{k} \mid X_{t}\right]\left(\rho y, \vec{\xi}_{n}\right)\right|^{p^{\prime}} d w_{\varphi}^{r}(y) \\
\leq & \int_{C^{r}}\left[2\left[\int_{0}^{t}\left\|\sigma_{s}\right\| d|\eta|(s)\right]^{k}\right]^{p^{\prime}} d w_{\varphi}^{r}(y)=2^{p^{\prime}}\left[\int_{0}^{t}\left\|\sigma_{s}\right\| d|\eta|(s)\right]^{k p^{\prime}}<\infty .
\end{aligned}
$$

Letting $\lambda \rightarrow-i q$ through $\mathbb{C}_{+}$, we have the result by the dominated convergence theorem.

Theorem 3.6. For $x \in C^{r}[0, t]$ let

$$
F(x)=\exp \left\{\int_{0}^{t} \theta(s, x(s)) d \eta(s)\right\} .
$$

Then under the assumptions given as in Theorem 3.5, $T_{q}^{(p)}\left[F \mid X_{t}\right]\left(y, \vec{\xi}_{n}\right)$ exists for $y \in C^{r}[0, t]$ and $\vec{\xi}_{n} \in \mathbb{R}^{(n+1) r}$, and it is given by

$$
T_{q}^{(p)}\left[F \mid X_{t}\right]\left(y, \vec{\xi}_{n}\right)=1+\sum_{k=1}^{\infty} \frac{1}{k !} T_{q}^{(p)}\left[F_{k} \mid X_{t}\right]\left(y, \vec{\xi}_{n}\right)
$$


where $T_{q}^{(p)}\left[F_{k} \mid X_{t}\right]\left(y, \vec{\xi}_{n}\right)$ is given as in Theorem 3.5.

Proof. By the Maclaurin series of the exponential function, we have

$$
F(x)=1+\sum_{k=1}^{\infty} \frac{1}{k !} F_{k}(x)
$$

and for $\lambda \in \mathbb{C}_{+}$or $\lambda=-i q, y \in C^{r}[0, t]$ and $\vec{\xi}_{n}=\left(\xi_{0}, \xi_{1}, \cdots, \xi_{n}\right) \in \mathbb{R}^{(n+1) r}$ we have

$$
\begin{aligned}
& 1+\sum_{k=1}^{\infty} \frac{1}{k !}\left|k ! \sum_{q_{1}+\cdots+q_{n}=k} \prod_{l=1}^{n} A\left(l, \lambda, y, \xi_{l-1}, \xi_{l}, q_{l}\right)\right| \\
\leq & 1+\sum_{k=1}^{\infty} \frac{1}{k !}\left[\int_{0}^{t}\left\|\sigma_{s}\right\| d|\eta|(s)\right]^{k}=\exp \left\{\int_{0}^{t}\left\|\sigma_{s}\right\| d|\eta|(s)\right\}
\end{aligned}
$$

where $A$ is given as in Theorems 3.2 and 3.5. Hence we have for $\lambda \in \mathbb{C}_{+}$

$$
T_{\lambda}\left[F \mid X_{t}\right]\left(y, \vec{\xi}_{n}\right)=1+\sum_{k=1}^{\infty} \frac{1}{k !} T_{\lambda}\left[F_{k} \mid X_{t}\right]\left(y, \vec{\xi}_{n}\right)
$$

since the convergence of (3.8) is uniform with respect to both $\lambda$ and $y$. For $1 \leq p \leq$ $\infty$ let $T_{q}^{(p)}\left[F \mid X_{t}\right]\left(y, \vec{\xi}_{n}\right)$ be given by the right hand side of (3.7). For $\lambda \in \mathbb{C}_{+}$we have

$$
\left|T_{\lambda}\left[F \mid X_{t}\right]\left(y, \vec{\xi}_{n}\right)-T_{q}^{(p)}\left[F \mid X_{t}\right]\left(y, \vec{\xi}_{n}\right)\right| \leq 2 \exp \left\{\int_{0}^{t}\left\|\sigma_{s}\right\| d|\eta|(s)\right\}
$$

and hence when $1<p \leq \infty$, for $\frac{1}{p}+\frac{1}{p^{\prime}}=1$ and $\rho>0$, we have

$$
\begin{aligned}
& \int_{C^{r}}\left|T_{\lambda}\left[F \mid X_{t}\right]\left(\rho y, \vec{\xi}_{n}\right)-T_{q}^{(p)}\left[F \mid X_{t}\right]\left(\rho y, \vec{\xi}_{n}\right)\right|^{p^{\prime}} d w_{\varphi}^{r}(y) \\
\leq & \int_{C^{r}}\left[2 \exp \left\{\int_{0}^{t}\left\|\sigma_{s}\right\| d|\eta|(s)\right\}\right]^{p^{\prime}} d w_{\varphi}^{r}(y)=2^{p^{\prime}} \exp \left\{p^{\prime} \int_{0}^{t}\left\|\sigma_{s}\right\| d|\eta|(s)\right\}<\infty .
\end{aligned}
$$

Letting $\lambda \rightarrow-i q$ through $\mathbb{C}_{+}$, we have the result by the dominated convergence theorem.

For $\nu \in \mathcal{M}\left(\mathbb{R}^{r}\right)$ define $\psi$ on $\mathbb{R}^{r}$ by

$$
\psi(\vec{u})=\int_{\mathbb{R}^{r}} \exp \{i\langle\vec{u}, \vec{v}\rangle\} d \nu(\vec{v}) .
$$

Then we have for $\vec{\xi}_{n}=\left(\xi_{0}, \xi_{1}, \cdots, \xi_{n}\right) \in \mathbb{R}^{(n+1) r}$ and $\lambda>0$

$$
\psi\left(y(t)+\lambda^{-\frac{1}{2}}(x(t)-[x](t))+\left[\vec{\xi}_{n}\right](t)\right)=\psi\left(y(t)+\xi_{n}\right)
$$


and

$$
\left|\psi\left(y(t)+\xi_{n}\right)\right| \leq\|\nu\| .
$$

By (3.10), (3.11), Theorems 3.2, 3.5 and 3.6, we have the following theorem.

Theorem 3.7. Let $G_{k}$ and $G$ be given by

$$
G_{k}(x)=F_{k}(x) \psi(x(t))
$$

and

$$
G(x)=F(x) \psi(x(t))
$$

for $x \in C^{r}[0, t]$, where $F_{k}, F$ and $\psi$ are given by (3.2), (3.6) and (3.9), respectively. Then, under the assumptions and notations given as in Theorems 3.2, 3.5 and 3.6, we have for $y \in C^{r}[0, t]$, nonzero real $q$ and $\vec{\xi}_{n}=\left(\xi_{0}, \xi_{1}, \cdots, \xi_{n}\right) \in \mathbb{R}^{(n+1) r}$

$$
T_{q}^{(p)}\left[G_{k} \mid X_{t}\right]\left(y, \vec{\xi}_{n}\right)=\psi\left(y(t)+\xi_{n}\right) T_{q}^{(p)}\left[F_{k} \mid X_{t}\right]\left(y, \vec{\xi}_{n}\right)
$$

and

$$
\begin{aligned}
T_{q}^{(p)}\left[G \mid X_{t}\right]\left(y, \vec{\xi}_{n}\right) & =\psi\left(y(t)+\xi_{n}\right) T_{q}^{(p)}\left[F \mid X_{t}\right]\left(y, \vec{\xi}_{n}\right) \\
& =\psi\left(y(t)+\xi_{n}\right)+\sum_{k=1}^{\infty} \frac{1}{k !} T_{q}^{(p)}\left[G_{k} \mid X_{t}\right]\left(y, \vec{\xi}_{n}\right) .
\end{aligned}
$$

\section{Remark 3.8.}

- If $F_{k}, G_{k}, F$ and $G$ are defined on $r$-dimensional Wiener space, then we can obtain the same results in Theorems $3.2,3.5,3.6$ and 3.7 with $\xi_{0}=\overrightarrow{0} \in \mathbb{R}^{r}$ in the expression of $\vec{\xi}_{n}$.

- If some of the $p_{l, j} \mathrm{~s}$ are in the set $\left\{t_{0}, t_{1}, \cdots, t_{n}\right\}$, we can obtain all the results in the present section with minor modifications.

- If $\eta=\mu+\sum_{l=1}^{n} \sum_{j=1}^{r_{l}} w_{l, j} \delta_{p_{l, j}}$ and some of the $r_{l} \mathrm{~s}$ are $\infty$, then, using the following version of the $\aleph_{0}$-nomial formula [7, p.41]

$$
\left(\sum_{p=0}^{\infty} b_{p}\right)^{n}=\sum_{h=0}^{\infty} \sum_{q_{0}+q_{1}+\cdots+q_{h}=n, q_{h} \neq 0} \frac{n !}{q_{0} ! q_{1} ! \cdots q_{h} !} b_{0}^{q_{0}} b_{1}^{q_{1}} \cdots b_{h}^{q_{h}},
$$

we can show that $T_{q}^{(p)}\left[G \mid X_{t}\right]\left(y, \vec{\xi}_{n}\right)$ exists in Theorem 3.7.

\section{Time-independent conditional Fourier-Feynman transform}

In the present section we evaluate the conditional analytic Fourier-Feynman 
transform of $G$ given $Y_{t}$, where $Y_{t}$ and $G$ are given by (2.4) and (3.12), respectively. For the purpose we need the following lemma.

Lemma 4.1. For $\lambda>0, \vec{v} \in \mathbb{R}^{r}, y \in C^{r}[0, t]$ and $\xi_{n-1} \in \mathbb{R}^{r}$ let

$$
\begin{aligned}
\Psi\left(n, \lambda, y, \xi_{n-1}, \vec{v}, \vec{v}_{n}, \vec{h}_{n}, \vec{s}_{n}\right)= & {\left[\frac{\lambda}{2 \pi\left(t-t_{n-1}\right)}\right]^{\frac{r}{2}} \int_{\mathbb{R}^{r}} D\left(n, y, \xi_{n-1}, \xi_{n}, \vec{v}_{n}, \vec{h}_{n}, \vec{s}_{n}\right) } \\
& \times \exp \left\{i\left\langle\xi_{n}, \vec{v}\right\rangle-\frac{\lambda}{2} \frac{\left\|\xi_{n}-\xi_{n-1}\right\|_{\mathbb{R}^{r}}^{2}}{t-t_{n-1}}\right\} d \xi_{n}
\end{aligned}
$$

where $\vec{v}_{n}, \vec{h}_{n}, \vec{s}_{n}$ and $D\left(n, y, \xi_{n-1}, \xi_{n}, \vec{v}_{n}, \vec{h}_{n}, \vec{s}_{n}\right)$ are given as in Theorem 3.2 with $l=n$. Then we have

$$
\begin{aligned}
& \Psi\left(n, \lambda, y, \xi_{n-1}, \vec{v}, \vec{v}_{n}, \vec{h}_{n}, \vec{s}_{n}\right) \\
= & \exp \left\{i\left\langle\xi_{n-1}, \vec{v}\right\rangle+i \sum_{u=0}^{r_{n}} \sum_{v=1}^{j_{u}+1}\left\langle y\left(s_{n, u, v}\right)+\xi_{n-1}, \vec{v}_{n, u, v}\right\rangle\right. \\
& \left.-\frac{t-t_{n-1}}{2 \lambda}\left\|\vec{v}+\sum_{u=0}^{r_{n}} \sum_{v=1}^{j_{u}+1} \frac{s_{n, u, v}-t_{n-1}}{t-t_{n-1}} \vec{v}_{n, u, v}\right\|_{\mathbb{R}^{r}}^{2}\right\} .
\end{aligned}
$$

Proof. For $\lambda>0$, we have by the change of variable theorem and (3.4)

$$
\begin{aligned}
& \Psi\left(n, \lambda, y, \xi_{n-1}, \vec{v}, \vec{v}_{n}, \vec{h}_{n}, \vec{s}_{n}\right) \\
= & {\left[\frac{\lambda}{2 \pi\left(t-t_{n-1}\right)}\right]^{\frac{r}{2}} \int_{\mathbb{R}^{r}} \exp \left\{i \sum _ { u = 0 } ^ { r _ { n } } \sum _ { v = 1 } ^ { j _ { u } + 1 } \left\langley\left(s_{n, u, v}\right)+\xi_{n-1}+\frac{s_{n, u, v}-t_{n-1}}{t-t_{n-1}}\left(\xi_{n}\right.\right.\right.} \\
& \left.\left.\left.-\xi_{n-1}\right), \vec{v}_{n, u, v}\right\rangle+i\left\langle\xi_{n-1}, \vec{v}\right\rangle+i\left\langle\xi_{n}-\xi_{n-1}, \vec{v}\right\rangle-\frac{\lambda}{2} \frac{\left\|\xi_{n}-\xi_{n-1}\right\|_{\mathbb{R}^{r}}^{2}}{t-t_{n-1}}\right\} d \xi_{n} \\
= & \exp \left\{i\left\langle\xi_{n-1}, \vec{v}\right\rangle+i \sum_{u=0}^{r_{n}} \sum_{v=1}^{j_{u}+1}\left\langle y\left(s_{n, u, v}\right)+\xi_{n-1}, \vec{v}_{n, u, v}\right\rangle-\frac{t-t_{n-1}}{2 \lambda} \| \vec{v}+\sum_{u=0}^{r_{n}}\right. \\
& \left.\sum_{v=1}^{j_{u}+1} \frac{s_{n, u, v}-t_{n-1}}{t-t_{n-1}} \vec{v}_{n, u, v} \|_{\mathbb{R}^{r}}^{2}\right\}
\end{aligned}
$$

which completes the proof.

Theorem 4.2. Let the assumptions and notations be given as in Theorem 3.2. Suppose that $Y_{t}$ is given by (2.4). Then for $\lambda \in \mathbb{C}_{+}, y \in C^{r}[0, t]$ and $\vec{\xi}_{n-1}=$ $\left(\xi_{0}, \xi_{1}, \cdots, \xi_{n-1}\right) \in \mathbb{R}^{n r}, T_{\lambda}\left[F_{k} \mid Y_{t}\right]\left(y, \vec{\xi}_{n-1}\right)$ exists and it is given by

$$
T_{\lambda}\left[F_{k} \mid Y_{t}\right]\left(y, \vec{\xi}_{n-1}\right)=k ! \sum_{q_{1}+\cdots+q_{n}=k}\left[\prod_{l=1}^{n-1} A\left(l, \lambda, y, \xi_{l-1}, \xi_{l}, q_{l}\right)\right] B\left(n, \lambda, y, \overrightarrow{0}, \xi_{n-1}, q_{n}\right)
$$


where for $\vec{v} \in \mathbb{R}^{r}, B\left(n, \lambda, y, \vec{v}, \xi_{n-1}, q_{n}\right)$ is given by the expression of $A\left(n, \lambda, y, \xi_{n-1}\right.$, $\left.\xi_{n}, q_{n}\right)$ replacing $D\left(n, y, \xi_{n-1}, \xi_{n}, \vec{v}_{n}, \vec{h}_{n}, \vec{s}_{n}\right)$ by $\Psi\left(n, \lambda, y, \xi_{n-1}, \vec{v}, \vec{v}_{n}, \vec{h}_{n}, \vec{s}_{n}\right)$ which is given by (4.1).

Proof. For $\vec{\xi}_{n-1}=\left(\xi_{0}, \xi_{1}, \cdots, \xi_{n-1}\right) \in \mathbb{R}^{n r}$, let $\left(\vec{\xi}_{n-1}, \xi_{n}\right)=\left(\xi_{0}, \xi_{1}, \cdots, \xi_{n-1}, \xi_{n}\right) \in$ $\mathbb{R}^{(n+1) r}$, where $\xi_{n} \in \mathbb{R}^{r}$. Then for $\lambda>0$ and $y \in C^{r}[0, t]$ we have by Theorem 3.2

$$
\begin{aligned}
K_{F_{k}}^{\lambda}(y, \vec{\xi})= & {\left[\frac{\lambda}{2 \pi\left(t-t_{n-1}\right)}\right]^{\frac{r}{2}} \int_{\mathbb{R}^{r}} I_{F_{k}}^{\lambda}\left(y,\left(\vec{\xi}_{n-1}, \xi_{n}\right)\right) \exp \left\{-\frac{\lambda\left\|\xi_{n}-\xi_{n-1}\right\|_{\mathbb{R}^{r}}^{2}}{2\left(t-t_{n-1}\right)}\right\} d \xi_{n} } \\
= & k !\left[\frac{\lambda}{2 \pi\left(t-t_{n-1}\right)}\right]^{\frac{r}{2}} \int_{\mathbb{R}^{r}}\left[\sum_{q_{1}+\cdots+q_{n}=k} \prod_{l=1}^{n} A\left(l, \lambda, y, \xi_{l-1}, \xi_{l}, q_{l}\right)\right] \\
& \times \exp \left\{-\frac{\lambda\left\|\xi_{n}-\xi_{n-1}\right\|_{\mathbb{R}^{r}}^{2}}{2\left(t-t_{n-1}\right)}\right\} d \xi_{n} \\
= & k ! \sum_{q_{1}+\cdots+q_{n}=k}\left[\prod_{l=1}^{n-1} A\left(l, \lambda, y, \xi_{l-1}, \xi_{l}, q_{l}\right)\right]\left[\frac{\lambda}{2 \pi\left(t-t_{n-1}\right)}\right]^{\frac{r}{2}} \\
& \times \int_{\mathbb{R}^{r}} A\left(n, \lambda, y, \xi_{n-1}, \xi_{n}, q_{n}\right) \exp \left\{-\frac{\lambda\left\|\xi_{n}-\xi_{n-1}\right\|_{\mathbb{R}^{r}}^{2}}{2\left(t-t_{n-1}\right)}\right\} d \xi_{n} \\
= & k ! \sum_{q_{1}+\cdots+q_{n}=k}\left[\prod_{l=1}^{n-1} A\left(l, \lambda, y, \xi_{l-1}, \xi_{l}, q_{l}\right)\right] B\left(n, \lambda, y, \overrightarrow{0}, \xi_{n-1}, q_{n}\right)
\end{aligned}
$$

where the last equality follows from Lemma 4.1. Now, by the Morera's theorem, we have the theorem.

For $\lambda \in \mathbb{C}_{+}$it is easy to prove

$$
\left|\Psi\left(n, \lambda, y, \xi_{n-1}, \vec{v}, \vec{v}_{n}, \vec{h}_{n}, \vec{s}_{n}\right)\right| \leq 1
$$

where $\Psi$ is given by (4.1). Applying the same method used in the proof of Theorems $3.5,3.6$ with (4.2), we can prove the following theorem.

Theorem 4.3. Let the assumptions and notations be given as in Theorems 3.2, 3.5, 3.6 and 4.2. Furthermore, let $q$ be a nonzero real number and $1 \leq p \leq \infty$. Then for $y \in C^{r}[0, t]$ and $\vec{\xi}_{n-1}=\left(\xi_{0}, \xi_{1}, \cdots, \xi_{n-1}\right) \in \mathbb{R}^{n r}, T_{q}^{(p)}\left[F_{k} \mid Y_{t}\right]\left(y, \vec{\xi}_{n-1}\right)$ and $T_{q}^{(p)}\left[F \mid Y_{t}\right]\left(y, \vec{\xi}_{n-1}\right)$ exist and they are given by

$$
\begin{aligned}
T_{q}^{(p)}\left[F_{k} \mid Y_{t}\right]\left(y, \vec{\xi}_{n-1}\right)= & k ! \sum_{\substack{q_{1}+\cdots+q_{n}=k\\
}}\left[\prod_{l=1}^{n-1} A\left(l,-i q, y, \xi_{l-1}, \xi_{l}, q_{l}\right)\right] \\
& \times B\left(n,-i q, y, \overrightarrow{0}, \xi_{n-1}, q_{n}\right)
\end{aligned}
$$

and

$$
T_{q}^{(p)}\left[F \mid Y_{t}\right]\left(y, \vec{\xi}_{n-1}\right)=1+\sum_{k=1}^{\infty} \frac{1}{k !} T_{q}^{(p)}\left[F_{k} \mid Y_{t}\right]\left(y, \vec{\xi}_{n-1}\right)
$$


Theorem 4.4. Under the assumptions and notations given as in Theorems 3.2, 3.7, 4.2 and 4.3, we have for $y \in C^{r}[0, t]$ and $\vec{\xi}_{n-1}=\left(\xi_{0}, \xi_{1}, \cdots, \xi_{n-1}\right) \in \mathbb{R}^{n r}$

$$
\begin{aligned}
T_{q}^{(p)}\left[G_{k} \mid Y_{t}\right]\left(y, \vec{\xi}_{n-1}\right)= & k ! \sum_{q_{1}+\cdots+q_{n}=k}\left[\prod_{l=1}^{n-1} A\left(l,-i q, y, \xi_{l-1}, \xi_{l}, q_{l}\right)\right] \\
& \times \int_{\mathbb{R}^{r}} B\left(n,-i q, y, \vec{v}, \xi_{n-1}, q_{n}\right) \exp \{i\langle y(t), \vec{v}\rangle\} d \nu(\vec{v})
\end{aligned}
$$

and

$$
\begin{aligned}
T_{q}^{(p)}\left[G \mid Y_{t}\right]\left(y, \vec{\xi}_{n-1}\right)= & \int_{\mathbb{R}^{r}} \exp \left\{i\left\langle y(t)+\xi_{n-1}, \vec{v}\right\rangle+\frac{t-t_{n-1}}{2 q i}\|\vec{v}\|_{\mathbb{R}^{r}}^{2}\right\} d \nu(\vec{v}) \\
& +\sum_{k=1}^{\infty} \frac{1}{k !} T_{q}^{(p)}\left[G_{k} \mid Y_{t}\right]\left(y, \vec{\xi}_{n-1}\right) .
\end{aligned}
$$

Proof. For $\vec{\xi}_{n-1}=\left(\xi_{0}, \xi_{1}, \cdots, \xi_{n-1}\right) \in \mathbb{R}^{n r}$, let $\left(\vec{\xi}_{n-1}, \xi_{n}\right)=\left(\xi_{0}, \xi_{1}, \cdots, \xi_{n-1}, \xi_{n}\right) \in$ $\mathbb{R}^{(n+1) r}$, where $\xi_{n} \in \mathbb{R}^{r}$. Then for $\lambda>0$ we have by Theorem 3.7

$$
\begin{aligned}
& K_{G_{k}}^{\lambda}\left(y, \vec{\xi}_{n-1}\right) \\
= & {\left[\frac{\lambda}{2 \pi\left(t-t_{n-1}\right)}\right]^{\frac{r}{2}} \int_{\mathbb{R}^{r}} I_{G_{k}}^{\lambda}\left(y,\left(\vec{\xi}_{n-1}, \xi_{n}\right)\right) \exp \left\{-\frac{\lambda\left\|\xi_{n}-\xi_{n-1}\right\|_{\mathbb{R}^{r}}^{2}}{2\left(t-t_{n-1}\right)}\right\} d \xi_{n} } \\
= & k !\left[\frac{\lambda}{2 \pi\left(t-t_{n-1}\right)}\right]^{\frac{r}{2}} \int_{\mathbb{R}^{r}} \psi\left(y(t)+\xi_{n}\right)\left[\sum_{q_{1}+\cdots+q_{n}=k} \prod_{l=1}^{n} A\left(l, \lambda, y, \xi_{l-1}, \xi_{l}, q_{l}\right)\right] \\
& \times \exp \left\{-\frac{\lambda\left\|\xi_{n}-\xi_{n-1}\right\|_{\mathbb{R}^{r}}^{2}}{2\left(t-t_{n-1}\right)}\right\} d \xi_{n} \\
= & k ! \sum_{q_{1}+\cdots+q_{n}=k}\left[\prod_{l=1}^{n-1} A\left(l, \lambda, y, \xi_{l-1}, \xi_{l}, q_{l}\right)\right]\left[\frac{\lambda}{2 \pi\left(t-t_{n-1}\right)}\right]^{\frac{r}{2}} \int_{\mathbb{R}^{r}} \exp \{i\langle y(t), \vec{v}\rangle\} \\
& \times \int_{\mathbb{R}^{r}} A\left(n, \lambda, y, \xi_{n-1}, \xi_{n}, q_{n}\right) \exp \left\{i\left\langle\xi_{n}, \vec{v}\right\rangle-\frac{\lambda\left\|\xi_{n}-\xi_{n-1}\right\|_{\mathbb{R}^{r}}^{2}}{2\left(t-t_{n-1}\right)}\right\} d \xi_{n} d \nu(\vec{v}) .
\end{aligned}
$$

Now by Theorem 3.2 and Lemma 4.1 we have

$$
\begin{aligned}
& {\left[\frac{\lambda}{2 \pi\left(t-t_{n-1}\right)}\right]^{\frac{r}{2}} \int_{\mathbb{R}^{r}} A\left(n, \lambda, y, \xi_{n-1}, \xi_{n}, q_{n}\right)} \\
& \times \exp \left\{i\left\langle\xi_{n}, \vec{v}\right\rangle-\frac{\lambda\left\|\xi_{n}-\xi_{n-1}\right\|_{\mathbb{R}^{r}}^{2}}{2\left(t-t_{n-1}\right)}\right\} d \xi_{n}=B\left(n, \lambda, y, \vec{v}, \xi_{n-1}, q_{n}\right)
\end{aligned}
$$


where $B\left(n, \lambda, y, \vec{v}, \xi_{n-1}, q_{n}\right)$ is given as in Theorem 4.2. We also have by (3.4)

$$
\begin{aligned}
& {\left[\frac{\lambda}{2 \pi\left(t-t_{n-1}\right)}\right]^{\frac{r}{2}} \int_{\mathbb{R}^{r}} \psi\left(y(t)+\xi_{n}\right) \exp \left\{-\frac{\lambda\left\|\xi_{n}-\xi_{n-1}\right\|_{\mathbb{R}^{r}}^{2}}{2\left(t-t_{n-1}\right)}\right\} d \xi_{n} } \\
= & {\left[\frac{\lambda}{2 \pi\left(t-t_{n-1}\right)}\right]^{\frac{r}{2}} \int_{\mathbb{R}^{r}} \exp \left\{i\left\langle y(t)+\xi_{n-1}, \vec{v}\right\rangle\right\} \int_{\mathbb{R}^{r}} \exp \left\{i\left\langle\xi_{n}-\xi_{n-1}, \vec{v}\right\rangle\right.} \\
& \left.-\frac{\lambda\left\|\xi_{n}-\xi_{n-1}\right\|_{\mathbb{R}^{r}}^{2}}{2\left(t-t_{n-1}\right)}\right\} d \xi_{n} d \nu(\vec{v}) \\
= & \int_{\mathbb{R}^{r}} \exp \left\{i\left\langle y(t)+\xi_{n-1}, \vec{v}\right\rangle-\frac{t-t_{n-1}}{2 \lambda}\|\vec{v}\|_{\mathbb{R}^{r}}^{2}\right\} d \nu(\vec{v}) .
\end{aligned}
$$

By the Morera's theorem and the dominated convergence theorem, we have the theorem.

\section{Remark 4.5.}

- If $F_{k}, G_{k}, F$ and $G$ are defined on $r$-dimensional Wiener space, then we can obtain the same results in Theorems 4.2, 4,3 and 4.4 with $\xi_{0}=\overrightarrow{0} \in \mathbb{R}^{r}$ in the expression of $\vec{\xi}_{n-1}$.

- If $\eta=\mu$ or $\eta=\sum_{l=1}^{n} \sum_{j=1}^{r_{l}} w_{l, j} \delta_{p_{l, j}}$, we can obtain more simple expressions in Theorems 4.2, 4.3 and 4.4.

- If some of the $p_{l, j} \mathrm{~s}$ are in the set $\left\{t_{0}, t_{1}, \cdots, t_{n}\right\}$, we can obtain all the results in the present section with minor modifications.

- If $\eta=\mu+\sum_{l=1}^{n} \sum_{j=1}^{r_{l}} w_{l, j} \delta_{p_{l, j}}$ and some of the $r_{l}$ s are $\infty$, then, using (3.13), we can show that $T_{q}^{(p)}\left[G \mid Y_{t}\right]\left(y, \vec{\xi}_{n-1}\right)$ exists in Theorem 4.4.

Acknowledgment. This work was supported by Kyonggi University Research Grant 2010 .

\section{References}

[1] R. H. Cameron and D. A. Storvick, Some Banach algebras of analytic Feynman integrable functionals, Lecture Notes in Mathematics 798, Springer, Berlin-New York, 1980.

[2] K. S. Chang, D. H. Cho and I. Yoo, Evaluation formulas for a conditional Feynman integral over Wiener paths in abstract Wiener space, Czechoslovak Math. J., 54(129)(2004), no. 1, 161-180.

[3] D. H. Cho, Conditional Fourier-Feynman transform and convolution product over Wiener paths in abstract Wiener space: an $L_{p}$ theory, J. Korean Math. Soc., 41(2004), no. 2, 265-294. 
[4] D. H. Cho, A simple formula for an analogue of conditional Wiener integrals and its applications, Trans. Amer. Math. Soc., 360(2008), no. 7, 3795-3811.

[5] D. H. Cho, A simple formula for an analogue of conditional Wiener integrals and its applications II, Czechoslovak Math. J., 59(134)(2009), no. 2, 431-452.

[6] M. K. Im and K. S. Ryu, An analogue of Wiener measure and its applications, J. Korean Math. Soc., 39(2002), no. 5, 801-819.

[7] G. W. Johnson and M. L. Lapidus, Generalized Dyson series, generalized Feynman diagrams, the Feynman integral and Feynman's operational calculus, Mem. Amer. Math. Soc., 62(1986), no. 351.

[8] H. H. Kuo, Gaussian measures in Banach spaces, Lecture Notes in Mathematics, 463, Springer-Verlag, Berlin-New York, 1975.

[9] K. S. Ryu and M. K. Im, A measure-valued analogue of Wiener measure and the measure-valued Feynman-Kac formula, Trans. Amer. Math. Soc., 354(2002), no. 12, 4921-4951.

[10] J. Yeh, Inversion of conditional expectations, Pacific J. Math., 52(1974), 631-640.

[11] J. Yeh, Inversion of conditional Wiener integrals, Pacific J. Math., 59(1975), no. 2, 623-638.

[12] J. Yeh, Transformation of conditional Wiener integrals under translation and the Cameron-Martin translation theorem, Tôhoku Math. J., 30(2)(1978), no. 4, 505-515. 